Published in final edited form as:

Lancet Haematol. 2019 June ; 6(6): e306-e316. doi:10.1016/S2352-3026(19)30050-X.

\title{
The Changing Burden of Late Health Outcomes in Adult Survivors of Childhood Acute Lymphoblastic Leukemia: A Report from the St. Jude Lifetime Cohort Study
}

\author{
Daniel A. Mulrooney, MD1,2,3, Geehong Hyun, PhD ${ }^{1}$, Kirsten K. Ness, PhD $^{1}$ [professor], \\ Nickhill Bhakta, MD ${ }^{1,4}$, Ching-Hon Pui, MD ${ }^{2,4}$ [professor], Matthew J. Ehrhardt, MD ${ }^{1,2}$, Kevin \\ R. Krull, PhD ${ }^{1,5}$ [professor], Deborah B. Crom, PhD², Wassim Chemaitilly, MD ${ }^{1,6}$, Deokumar \\ Srivastava, $\mathrm{PhD}^{7}$ [professor], Mary V. Relling, PharmD ${ }^{8}$ [professor], Sima Jeha, MD $^{2,4}$ \\ [professor], Daniel M. Green, MD ${ }^{1,2}$ [professor], Yutaka Yasui, PhD ${ }^{1}$ [professor], Leslie L. \\ Robison, $\mathbf{P h D}^{1}$ [professor], Melissa M. Hudson, $\mathbf{M D}^{1,2,3}$ [professor] \\ ${ }^{1}$ Department of Epidemiology and Cancer Control, St. Jude Children's Research Hospital, \\ Memphis, TN \\ ${ }^{2}$ Department of Oncology, St. Jude Children's Research Hospital, Memphis, TN \\ ${ }^{3}$ Departments of Pediatrics and Medicine, University of Tennessee Health Science Center, \\ College of Medicine, Memphis, TN \\ ${ }^{4}$ Department of Global Pediatric Medicine, St. Jude Children's Research Hospital, Memphis, TN \\ ${ }^{5}$ Department of Psychology, St. Jude Children's Research Hospital, Memphis, TN \\ ${ }^{6}$ Department of Pediatric Medicine, St. Jude Children's Research Hospital, Memphis, TN \\ ${ }^{7}$ Department of Biostatistics, St. Jude Children's Research Hospital, Memphis, TN \\ ${ }^{8}$ Department of Pharmaceutical Sciences, St. Jude Children's Research Hospital, Memphis, TN
}

\section{Summary}

Background: Treatment for childhood acute lymphoblastic leukemia (ALL) has changed over the last five decades but the impact of these changes on late health outcomes has not been assessed. We aimed to characterize the magnitude of morbidity and patterns of health outcomes among survivors of ALL treated over time.

\footnotetext{
Address for correspondence: Daniel A. Mulrooney, MD, MS, Division of Cancer Survivorship, Department of Oncology, St. Jude Children's Research Hospital, MS 735, 262 Danny Thomas Place, Memphis, TN 38105. Telephone: (901)-595-5847; Fax: (901)-595-5845; daniel.mulrooney@stjude.org.

Contributions

DAM, KKN, WC, DKS, LLR, MMH contributed to study design

LLR, MMH contributed to funding

DAM, KKN, MJE, DBC, WC, MJE, LLR, MMH contributed to data collection

DAM, GH, KKN, NB, CHP, KRK, WC, DKS, YY, LLR contributed to data analysis

DAM, GH, KKN, NB, CHP, MJE, KRK, DBC, WC, DKS, MVR, SJ, DMG, YY, LLR, MMH contributed to data interpretation

DAM, GH, KKN, CHP, MJE, KRK, DMG, YY, MMH contributed to writing the manuscript

DAM, GH, KKN, NB, CHP, KRK, WC, DKS, MVR, SJ, DMG, YY, LLR, MMH contributed to approval of the manuscript

Declaration of Interests

MVR reports grants from Sigma Tau/Shire/Baxalta during the conduct of the study. All other authors declare no conflicts of interest. Data Sharing: Data are shared according to the SJLIFE Cohort Study Resource Sharing Plan.
} 
Methods: History/physical examination, laboratory analysis, physical fitness, and neurocognitive testing were performed in 980 adult survivors ( $\geq 18$ years of age, $\geq 10$ years from diagnosis) of pediatric ALL and 272 age-, frequency matched community controls participating in the St. Jude Lifetime Cohort Study. Health conditions were graded per a modified version of the Common Terminology Criteria for Adverse Events. Neurocognitive domains of attention and executive function were measured, and age-adjusted z-scores calculated and compared to national norms and controls. Mean cumulative count was used to determine the age-standardized cumulative burden of health conditions over time.

Findings: Survivors [50\% female, median age at diagnosis 5 years (range $0 \cdot 2-19 \cdot 5$ ), median of 30.0 years $(11.9-51.8)$ from diagnosis] were $35.8(19 \cdot 6-63.4)$ years at evaluation compared to $35 \cdot 1(18 \cdot 3-71 \cdot 1)$ years for controls. Survivors had significantly more, growth hormone deficiency, hypogonadism, and neuropathy than controls. By age 30, ALL survivors experienced, on average, $5 \cdot 4$ grade $1-4$ conditions (95\% CI 5.1-5.8) including 3.2 grade $2-4$ conditions (95\% CI 2.9-3.4) compared to 2.0 grade $1-4$ (95\% CI 1.7-2.2) and 1.2 grade $2-4$ (95\% CI 1.03-1.4) conditions among controls. The cumulative burden of grade $2-4$ conditions involved multiple organ systems for survivors treated on protocols between 1962-1991 but following elimination of cranial irradiation are now predominately musculoskeletal and endocrine disorders for those on protocols between 1991-2007.

Interpretation: While changes in ALL therapy have improved overall survival, the burden of late morbidity remains high. However, the pattern of late toxicities has markedly changed over time, with survivors experiencing a reduction in conditions that are life-threatening. Maintaining health and quality of life for survivors requires continued medical surveillance, counseling, and lifestyle modifications.

\section{Introduction}

Treatment for childhood acute lymphoblastic leukemia (ALL) has evolved over the last five decades and transformed a fatal disease into one with five-year survival rates in excess of 90\%. ${ }^{1}$ Children successfully treated for ALL represent approximately $16 \%$ of the long-term survivors of childhood cancer in the U.S., estimated to surpass 500000 by the end of $2019 .{ }^{2}$

While it is well-recognized that adult survivors of childhood ALL are at increased risk of chronic health conditions, subsequent neoplasms, neurocognitive impairment, and premature mortality, ${ }^{3}$ studies have generally not characterized the overall health burden in large systematically evaluated populations or described how evolution in therapy has impacted long-term health. In the largest clinical cohort of prospectively and systematically evaluated survivors of childhood ALL to date, we provide a precise characterization of the magnitude of late-morbidity and changing patterns of late health outcomes among survivors treated over four decades.

\section{Methods}

\section{Study Population}

The St. Jude Lifetime (SJLIFE) cohort is a study with prospective follow-up and ongoing data accrual designed to facilitate longitudinal clinically-based evaluation of health 
outcomes among survivors of pediatric malignancies. ${ }^{4}$ Survivors included in this analysis were treated for pediatric ALL at St. Jude Children's Research Hospital (SJCRH), $\geq 18$ years old, $\geq 10$ years from initial cancer diagnosis, and had completed an initial on-campus SJLIFE evaluation. Cumulative chemotherapy and radiation dose exposures and major medical events during and after therapy were abstracted from the medical records.

Participants with Down syndrome ( $\mathrm{n}=\mathrm{six})$ or those treated with stem cell transplant $(\mathrm{n}=23)$ were not included in the current analysis. The SJLIFE evaluation included physical examination; a core laboratory assessment; physical and neurocognitive performance evaluations; questionnaires detailing demographic, medical history, quality of life, and health habits; and risk-based assessments per the Children's Oncology Group's Long-Term Follow-Up Guidelines for Survivors of Childhood, Adolescent, and Young Adult Cancers. ${ }^{5}$ A frequency matched control group was recruited from friends and non-first degree relatives of current and former SJCRH patients (i.e. individuals who might be accompanying or visiting a patient), St. Jude employees, or advertising within the Memphis area, minimizing the chance of close relatives of SJLIFE participants being a control participant. Informed consent was obtained from all participants as well as for release of outside medical records to validate and grade conditions diagnosed prior to or after the SJLIFE study assessment. The protocol was approved by the SJCRH Institutional Review Board.

\section{Chronic Health Conditions}

One hundred eleven health conditions, grouped by organ system, were defined and graded per a modified version of the National Cancer Institute's Common Terminology Criteria for Adverse Events (CTCAE) version 4.03. ${ }^{6}$ Chronic disease burden was determined from the mean cumulative count of recurrent/multiple health events over time, as previously described. ${ }^{7}$ Prevalence was assessed for conditions most commonly reported among ALL survivors including: cardiovascular (cardiomyopathy, hypertension, hypercholesterolemia, and hypertriglyceridemia), endocrine/reproductive (growth hormone deficiency, adrenal insufficiency, hypothyroidism, hypogonadism, oligospermia/azoospermia, impaired glucose metabolism, overweight/obesity), bone mineral density, osteonecrosis, neurologic (peripheral motor and sensory neuropathy, stroke), ocular (cataracts), and subsequent neoplasms.

\section{Attention and Executive Function}

Neurocognitive testing focused on the domains of attention and executive function. Measures of attention included Trail Making Test Part $\mathrm{A}^{8}$ (focused attention) and Conner's Continuous Performance Test-II ${ }^{9}$ (sustained/variable attention). Measures of executive function included Trail Making Test Part B ${ }^{8}$ (cognitive flexibility), Controlled Oral Word Association Test $^{8}$ (verbal fluency), and Wechsler Adult Intelligence Scale-III Digit Span Test backward ${ }^{10}$ (working memory). Using normative data provided by the individual test developers, age-adjusted z-scores were calculated for survivors and controls such that the population reference has a mean of zero and a standard deviation of one, consistent with clinical standards. 


\section{Physical Function}

Aerobic function was assessed using the six minute walk test $(6 \mathrm{MWT})^{11}$ and mobility with the Timed-Up-and-Go (TUG) test. ${ }^{12}$ Abnormal tests were defined as walking $\leq 490$ meters in six minutes and taking more than six seconds to rise from a chair, walk three meters, return, and sit.

\section{Statistical Analyses}

Descriptive statistics were generated to characterize study participants. Two-sample t-tests and $\chi^{2}$ statistics were used to compare demographic and treatment-related characteristics between participants and nonparticipants. The mean number of recurrent/multiple health events experienced over time (mean cumulative count) was used to calculate the cumulative burden of grade 2-4 chronic disease adjusting for competing risks. ${ }^{13}$ The age-specific cumulative burden and $95 \%$ confidence intervals, overall and by organ system, were estimated by summarizing chronic health conditions occurring per ALL survivor and community control using the bootstrap percentile method. ${ }^{14}$ St. Jude Total Therapy protocols were grouped to reflect major therapeutic changes over the last half century (appendix p. 1). ${ }^{15}$ Cumulative burden of chronic disease was estimated for each of the five protocol strata [Total protocols II-IX (1962-1979), X (1979-1983), XI-XII (1984-1991), XIII-XIV (1991-1999), and XV (2000-2007)], using a method of inverse probability of treatment weighting to adjust for differences in the age distribution at the last follow-up, and their differences were tested using permutation test. Seventy-two (7.4\%) survivors were treated according to, but not enrolled on, a Total Therapy protocol and were excluded from protocol stratified analyses.

The standardized incidence ratios of subsequent neoplasms used sex-, age-, race-, and calendar-year specific cancer incidence from the Surveillance, Epidemiology, and End Results (SEER) data from the National Cancer Institute. Person-time at risk for subsequent neoplasms was calculated from one year after the diagnosis of ALL until diagnosis of subsequent neoplasm, death, or last follow-up.

Associations between cumulative burden and neurocognitive function and physical performance were evaluated using age-adjusted z-scores or percentages and relative odds, as appropriate, and compared using t-tests, $\chi^{2}$ statistics, and unconditional multivariable logistic regression models, adjusted for sex, age at diagnosis, educational attainment, employment, health insurance status. SAS version 9.4 and $\mathrm{R}$ version 3.4 .3 were used for all statistical analyses.

The study is registered at ClinicalTrials.gov, Identifier: .

\section{Role of the funding source}

The funders of the study had no role in the study design; data collection, analysis, or interpretation; or in writing of the report. The corresponding author had full access to all of the data in the study and final responsibility for the decision to submit for publication. 


\section{Results}

Of 1456 survivors of ALL eligible for participation in the SJLIFE cohort, 980 (67.3\%) had completed an evaluation on the SJCRH campus as of June 30, 2015. An additional 67 (4.6\%) agreed to participate by survey only and 109 (7.5\%) had a pending assessment. Of the remaining non-participants, $52(3.6 \%)$ could not be located and 247 (17\%) declined to participate (Figure 1). Participating survivors differed from non-participants by gender $(\mathrm{p}=0.044)$ and race $(\mathrm{p}=0.017)$ (appendix p. 3).

Survivors were evenly divided by gender, median age at diagnosis was five years (IQR 3.1$9 \cdot 1$ years), and median time from diagnosis was 30.0 years (22.7-36.3) (Table 1). Median age at evaluation was 35.8 years $(29.4-42.9)$ for survivors and $35.1(28.7-42.6)$ for controls $(\mathrm{p}=0 \cdot 30)$. Most ALL survivors (592 of the $980[60 \cdot 4 \%]$ ) had attended some college but only $307(31.3 \%)$ attained a degree compared to $202(74.3 \%)$ and $118(43.4 \%)$, respectively, of 272 controls $(\mathrm{p}<0 \cdot 0001)$. Four hundred eighty-eight survivors $(49.8 \%)$ were employed fulltime compared to $172(63.2 \%)$ controls (107 [10.9\%] survivors and 32 [11.8\%] controls were employed part-time) $(\mathrm{p}<0 \cdot 0001)$.

All survivors were enrolled on $(n=908)$ or treated per $(n=72)$ one of the 15 Total Therapy protocols between 1962 and 2007. As expected, treatment varied over time, particularly with central nervous system prophylaxis (appendix p. 1). Nearly all (943 of 980 survivors [96.2\%]) received intra-thecal chemotherapy, 556 (56.7\%) received cranial and/or cranialspinal (CRT) radiation (329 [33.6\%] $24 \mathrm{~Gy}$ and 227 [23.2\%] $18 \mathrm{~Gy})$, and $424(43.3 \%)$ had no radiation exposure. High dose methotrexate (HDMTX) was given to $618(63.1 \%)$ and, in particular to 409 (96.5\%) of the 424 non-irradiated survivors.

\section{Chronic Health Conditions}

As expected, after adjusting for gender, age at evaluation, and race, the prevalence and severity of chronic health conditions was elevated across most organ systems, particularly endocrine, reproductive, and neurologic, compared to controls (Table 2). There was no statistically significant difference in cardiomyopathy or dyslipidemia. The prevalence of hypertension was higher among survivors compared to controls (53.8\% [527 of 980 survivors] vs. $43.7 \%$ [ 119 of 272 controls], $\mathrm{p}=0 \cdot 0.84$ ), though not statistically significant, with 180 survivors $(18.4 \%) \geq$ grade 2 . There was no statistically significant increase in the number of overweight/obese survivors (71.8\% [704] vs. 63.3\% [172] of controls, $\mathrm{p}=0.13)$ or in the prevalence of impaired glucose metabolism (19.5\% [190] vs. 19.9\% [54], $\mathrm{p}=0.26)$ compared to controls.

One hundred eighty (18.4\%) survivors had 626 subsequent neoplasms (123 benign meningiomas or non-invasive non-melanoma skin cancers, 503 malignant). All but four of the $180(2.0 \%)$ survivors had prior radiation exposure. Standardized incidence ratios (SIR) by cancer site and type are shown in appendix pp. $4-5$, as well as by radiation exposure and CTCAE grades. Overall, ALL survivors had a higher incidence of a subsequent neoplasm (SIR 1.2, 95\% CI 1.03-1.4). The incidence was significantly elevated for central nervous system (CNS) malignancies (SIR 23.3 95\% CI 19.6-27.5) and thyroid cancers (3.9 95\% CI $2 \cdot 4-6 \cdot 0)$. 
The cumulative burden of grade 1-4 and 2-4 chronic conditions was elevated among survivors compared to controls across all age groups and organ systems (appendix pp. 6-7). By age 30 years, an individual ALL survivor had, on average 5.4 grade $1-4$ conditions (95\% CI 5.1-5.8) and 3.2 grade 2-4 conditions (95\% CI 2.9-3.4), compared to 2.0 (95\% CI $1.7-$ $2 \cdot 2$ ) and $1 \cdot 2$ (95\% CI 1.03-1.4) among controls (Figure 2).

\section{Temporal trends in late morbidity}

The 30-year age standardized cumulative burden of grade 2-4 conditions differed across treatment eras (appendix p. 8), decreasing from Total II-IX to Total X (0.42/person 95\% CI $0.032-0.81)$ and increasing again by Total XV (0.69/person 95\% CI 0.11-1.26). Importantly, the organ systems impacted changed substantially over time (Figures $3 \& 4$ ). Survivors treated between 1962-1991, on protocols in which most patients received prophylactic CRT, had grade 2-4 conditions affecting multiple organ systems, including subsequent malignancies, neurologic sequelae, endocrinopathies, and infectious complications among others. As treatment evolved, with the increased utilization of CNS-active systemic therapy (dexamethasone and asparaginase) and intensified intrathecal chemotherapy, the type of organ dysfunction changed (appendix pp. 9-12). Stroke and seizures, likely a complication of CRT, made up much of the neurologic burden on early protocols ( 2.4 and 16.4, respectively, per 100 survivors treated on Total II-IX) but have largely been replaced by peripheral neuropathies, both motor and sensory ( 13.3 and 6.4, respectively, per 100 survivors on Total XV). Endocrinopathies among survivors treated on protocols II-XII largely involved adrenal insufficiency and growth hormone deficiency (likely due to CRTinduced hypothalamic pituitary dysfunction) and hypothyroidism, while impaired glucose metabolism and obesity became more prominent among those treated on more recent protocols. Impaired glucose metabolism increased from 3.7 per 100 survivors treated on protocols II-IX to 5.4 per 100 survivors treated on Total XV. Obesity rates were low for survivors of the earliest trials but increased steadily with time reaching 59.4 per 100 survivors of Total XV. Musculoskeletal late effects were rare among survivors of earlier protocols becoming progressively more prominent, particularly for those treated on Total XIII and beyond. This was driven by changes in decreased bone mineral density from 0 to $10 \cdot 2$ per 100 survivors and osteonecrosis from 0 to 63.5 per 100 survivors treated on Total II-IX and Total XV, respectively; likely due to intensified use of asparaginase and the replacement of prednisone with dexamethasone. Notably, infectious complications, particularly hepatitis $\mathrm{C}$ infections, decreased across the years, likely due to improved blood product screening, management of neutropenia, and supportive care measures.

\section{Temporal trends in physical function}

Figure 5 shows the frequency of impaired aerobic function and mobility, adjusted for current age, gender, body mass index, and neuropathy, by treatment protocol and stratified by radiation exposure. Overall, compared to controls, nearly twice as many ALL survivors had impaired aerobic function ( $23.3 \%$ [217 of 930 who completed the 6MWT] vs. $12.5 \%$ [34 of 271 completing the test) and mobility (37.6\% [359 of 954 completing the TUG test) vs. $18.4 \%$ [50 of 272 completing the test). Impairment was increased across all protocols, regardless of CNS prophylaxis therapy. Survivors on Total XV, treated without radiation, had similar aerobic dysfunction as irradiated survivors on Total XIII-XIV ( 9 of 30 survivors, 
[30\%]). Decreased mobility persisted across all protocols with nearly half (12 of 30 [46.5\% after age-standardization and adjustment for age at evaluation, gender, BMI, and neuropathy]) of the most recently treated Total XV survivors showing impairment.

\section{Temporal trends in neurocognition}

Attention and executive function for ALL survivors improved as treatment protocols restricted and eventually eliminated CRT (Figure 6). Among non-irradiated survivors, attention did not differ from population normative data or controls, with the exception of lower focused attention compared to controls. Improvements were also seen in executive function, however, those treated without CRT continued to have deficits compared to both the general population and controls.

\section{Health Burden on Neurocognitive and Physical Function}

The number of chronic health conditions was directly associated with neurocognitive and physical performance (appendix p. 13). Age-adjusted z-scores for attention and memory decreased as the number of conditions increased. While the impact on sustained attention was not significant, decreases were noted in variable and focused attention. Impaired executive function also increased directly with the number of chronic health conditions, with the most significant impact on cognitive flexibility. Similarly, the odds of an abnormal 6MWT and impaired mobility increased with the number of chronic conditions ( $\mathrm{p}_{\text {trend }} \leq$ 0.0001). More than three-fourths of those with 5-6 conditions and impaired aerobic function (54 of 69) and mobility ( 89 of 110), and >90\% (42 of 44 impaired aerobic function and 62 of 69 impaired mobility) with 7-12 conditions had been exposed to CRT (appendix p. 14).

\section{Discussion}

The evolution of therapy for pediatric ALL over the last half century has not only increased survival rates but also enhanced the understanding of late health outcomes following cytotoxic therapy. Recognition of treatment-related sequelae has led to therapeutic modifications that have reduced, but not eliminated, late adverse events. In this large systematically evaluated cohort, we describe not only the prevalence but also, importantly, the magnitude of chronic health conditions among adult survivors of childhood ALL and assessed their impact on physical and cognitive function. Not only did nearly all survivors (99\%) have at least one chronic health condition but applying the cumulative burden metric, the actual number of conditions per individual survivor was essentially double the number identified in community controls. Understanding the multiplicity of late health events among ALL survivors provides a more robust assessment of the morbidity experienced by these individuals and, for the first time to our knowledge, identifies changing patterns in late outcomes across treatment eras.

The health conditions identified, particularly in the endocrine, reproductive, neurologic, and cardiovascular systems are similar to other reports but in greater magnitude. Among ALL survivors ( $\mathrm{n}=2$ 599) surveyed in the multi-institutional Childhood Cancer Survivor Study (CCSS), 50\% reported one or more chronic health conditions compared to $37.8 \%$ in a sibling comparison group. ${ }^{3}$ The most prevalent conditions were endocrine (4.4\%), cardiac 
(3.2\%), and neurologic (2.4\%). Systematic clinical assessments performed in the SJLIFE cohort identified abnormalities in many of the same organ systems but at a higher prevalence and across all CTCAE grades.

Identification of conditions at an earlier stage has implications for clinical management and preservation of health. Initial management of many of the identified conditions typically begins with health counseling and lifestyle modification. For example, over half of the survivors had evidence of hypertension, while a third (35.4\%) were pre-hypertensive (grade 1 ), and $18.4 \%$ were more severe (grades $2-3$ ), requiring medical intervention. While a high prevalence of hypertension, compared to the general population, has been reported among childhood cancer survivors ${ }^{16}$, our study is, to our knowledge, the first to include prehypertension, the precursor to incident hypertension and a marker of cardiovascular risk. Recently re-classified as elevated blood pressure (120-129/<80) or Stage 1 hypertension (130-139/80-89) by the Joint National Committee on Prevention, Detection, Evaluation, and Treatment of High Blood Pressure ${ }^{17}$, pre-hypertension may require intervention depending upon risk assessments. More aggressive intervention is suggested for those with pre-existing cardiovascular disease and/or an elevated atherosclerotic cardiovascular disease (ASCVD) risk. In our young adult population, an elevated ASCVD risk is unlikely, however, hypertension is a significant modifier of anthracycline-induced cardiomyopathy, increasing the risk nearly 86-fold among survivors with hypertension and anthracycline exposure. ${ }^{18}$ While the overall prevalence of cardiomyopathy in our study was low (2.7\%), likely due to the lower mean dose exposure to anthracyclines $\left(105 \mathrm{mg} / \mathrm{m}^{2}\right)$ in the Total Therapy protocols, monitoring and early management of pre-hypertension and hypertension may be warranted to preserve cardiovascular health. Other conditions, such as low bone mineral density, would also benefit from lifestyle changes but require additional screening and potential treatment of associated conditions, such as sex steroid deficiencies. The number and severity of chronic conditions in cancer survivors has been associated with poor health-related quality of life, more so than the original cancer diagnosis and treatment. ${ }^{19}$ Preventive care, established early for this population, may help reduce or ameliorate the high prevalence of chronic health conditions.

Despite changes in therapy over time, the overall magnitude of adverse health outcomes experienced by survivors of ALL has not decreased. Survivors of childhood ALL continue to experience on average over five chronic health conditions and, importantly, three of grade 24 severity, compared to 1.96 and 1.21 , respectively, among controls. Importantly, as survivors age these numbers increase at rates nearly double that in the control population. It is encouraging that the pattern of health conditions has changed with a reduction in incidence of conditions that are immediately life-threatening. Survivors treated on older protocols (Total II-XII, 1962-1979) experienced late effects involving multiple organ systems (reproductive, neurologic, infectious, and gastrointestinal), while those treated more recently (Total XIII-XIV and Total XV, 1991-2007) had more musculoskeletal effects, likely related to more intensive use of dexamethasone and asparaginase. Screening children treated on Total XV for osteonecrosis, Kaste et al. ${ }^{20}$ found 40 extensive osteonecrotic lesions among patients $\geq 10$ years old, none of which improved with time and 19 required surgical intervention. In genome-wide association studies adjusting for age and treatment, multiple genetic variants were associated with grade $3-4$ osteonecrosis. ${ }^{21}$ Pharmacogenomics may 
lead to earlier identification of patients at risk and guide development of preventive measures.

The cumulative burden involving the endocrine system has persisted over time, although the specific types of endocrinopathies have changed. Survivors treated in earlier eras have endocrinopathies related to hypothalamic pituitary axis dysfunction, due to use of CRT for CNS treatment and/or prophylaxis. As these treatments changed over the years, hypothalamic dysfunction decreased but was replaced by impairments in glucose metabolism and body composition. Probably due to the national trend of increased weight in the general population, the higher prevalence of overweight/obesity among ALL survivors ( $71.8 \%$ vs. $63.3 \%, \mathrm{p}=0.13$ ) compared to our community control population did not meet statistical significance. Changes in endocrine dysfunction may impact other late cancerrelated sequelae as well as have implications for long-term health management.

Reductions in CRT have fortunately reduced the risk of stroke and seizures among ALL survivors but have been replaced by increased sensory and motor neuropathies that may impair physical activity, as suggested by the lack of improvement in aerobic function and mobility over treatment protocols. Others have identified similar deficits among ALL survivors, despite elimination of CRT, and found associations between muscle strength and a variety of chemotherapy exposures. ${ }^{22}$ While the evidence for exercise interventions in children during cancer therapy is mixed ${ }^{23}$, the benefits of increased physical activity have been shown in many adult onset cancers. ${ }^{24}$ Overcoming barriers and promoting healthy lifestyles among cancer survivors requires a multidisciplinary approach involving primary care providers and ancillary medical services, such as physical therapy, to ensure programs structured to address the unique needs of these individuals.

Improvements in cognitive function with reductions in the intensity of CNS prophylactic therapies have been shown by other investigators. While some studies, with shorter followup, did not identify dysfunction among those not exposed to $\mathrm{CRT}^{25}$, most have shown reductions but persistence of deficits, even among those treated with chemotherapy only. ${ }^{26}$ Focusing on measures of attention and executive function, our study has the longest followup (median $=30$ years) and largest number of ALL survivors not treated with CRT $(n=424)$. Improvements were evident with reductions in CRT, and attention deficits no longer differed from population norms or controls. However, executive function, even among those not exposed to CRT, continued to fall below normative values and controls. In an earlier report from the SJLIFE cohort, Krull et al. ${ }^{27}$ found nearly $16 \%$ of those not exposed to CRT had evidence of severe executive dysfunction at a mean of 26 years from diagnosis. Furthermore, executive dysfunction has been associated with plasma methotrexate concentrations, structural changes in the brain cortex, and increased activity in the frontal lobes. ${ }^{28}$ In the only other study with follow-up similar to ours (mean=25 years), Schuitema and colleagues ${ }^{29}$ used magnetic resonance imaging to study fractional anisotropy, a measure of white matter microstructure. Compared to controls, significant decreases in white matter integrity were identified among irradiated survivors $(n=44)$, with a trend noted among those treated with chemotherapy only $(n=49)$, in a distribution associated with executive dysfunction. Continued follow-up is needed to understand the trajectory of cognitive impairment among ALL survivors, exposed or unexposed to CRT, as they age. 
Despite the longest follow-up to date among the largest number of adult survivors of childhood ALL systematically and comprehensively assessed, a number of limitations should be considered when interpreting our results. It is possible that improved survival rates and increased screening have led to a degree of surveillance bias, limiting our ability to assess an overall decline in the burden of health conditions. Additionally, participation required returning to the SJCRH campus for a medical evaluation, potentially excluding individuals unable to return due to other commitments, health limitations, or death, thus, over- or under-estimating the prevalence of chronic health conditions. While $67.3 \%$ of eligible ALL survivors returned and selection bias could play a role, significant differences between SJLIFE participants and non-participants have not been identified. ${ }^{30}$ Comparisons of the cumulative burden of a specific single condition across different protocols requires caution. Due to the rarity of some of the conditions and/or small differences across groups, the statistical power for testing across-protocol comparisons of a single condition is limited. Additionally, not all participants completed all physical and neurocognitive tests. The missing data were minimal $(<6 \%)$, and we assumed this to be non-informative and missing at random. Only those participants with evaluable studies for a particular outcome were included in the analysis of that outcome.

Fifty years of clinical research implemented across the Total Therapy program has profoundly changed the outcomes of children diagnosed with ALL. Lessons learned from tested therapies have resulted in a population of long-term survivors with a different pattern of treatment-related sequelae. While the character of late health outcomes has evolved, the impact on future health remains, requiring ongoing surveillance and counseling to ensure preservation of health across the life span.

\section{Supplementary Material}

Refer to Web version on PubMed Central for supplementary material.

\section{Acknowledgments}

Supported by Cancer Center Support (CORE) Grant (CA21765) to St. Jude Children's Research Hospital (PI: Dr. Charles W. Roberts), U01 CA195547 (MPI: Drs. Melissa M. Hudson and Leslie L. Robison), P50 GM 115279 (PI: Dr. Mary V. Relling), and the American Lebanese Syrian Associate Charities (ALSAC), Memphis, TN.

Funding: US National Cancer Institute and the American Lebanese Syrian Associate Charities.

\section{References}

1. Pui CH, Campana D, Pei D, et al. Treating childhood acute lymphoblastic leukemia without cranial irradiation. N Engl J Med 2009; 360(26): 2730-41. [PubMed: 19553647]

2. Robison LL, Hudson MM. Survivors of childhood and adolescent cancer: life-long risks and responsibilities. Nat Rev Cancer 2014; 14(1): 61-70. [PubMed: 24304873]

3. Mody R, Li S, Dover DC, et al. Twenty-five-year follow-up among survivors of childhood acute lymphoblastic leukemia: a report from the Childhood Cancer Survivor Study. Blood 2008; 111(12): 5515-23. [PubMed: 18334672]

4. Hudson MM, Ness KK, Nolan VG, et al. Prospective medical assessment of adults surviving childhood cancer: study design, cohort characteristics, and feasibility of the St. Jude Lifetime Cohort study. Pediatr Blood Cancer 2011; 56(5): 825-36. [PubMed: 21370418] 
5. Landier W, Bhatia S, Eshelman DA, et al. Development of risk-based guidelines for pediatric cancer survivors: the Children's Oncology Group Long-Term Follow-Up Guidelines from the Children's Oncology Group Late Effects Committee and Nursing Discipline. J Clin Oncol 2004; 22(24): 4979_ 90. [PubMed: 15576413]

6. Hudson MM, Ehrhardt MJ, Bhakta N, et al. Approach for Classification and Severity Grading of Long-term and Late-Onset Health Events among Childhood Cancer Survivors in the St. Jude Lifetime Cohort. Cancer Epidemiol Biomarkers Prev 2017; 26(5): 666-74. [PubMed: 28035022]

7. Bhakta N, Liu Q, Ness KK, et al. The cumulative burden of surviving childhood cancer: an initial report from the St Jude Lifetime Cohort Study (SJLIFE). Lancet 2017; 390(10112): 2569-82. [PubMed: 28890157]

8. Strauss E, Sherman EM, Spreen O. A Copendium of Neuropsychological Tests: Administration, Norms, and Commentary. . Third Edition ed: Oxford University Press 2006.

9. Conners CK. Conners' Continous Performance Test II Multi-Healht Systems 2001.

10. Wechsler D Wechsler Adult Intelligence Scale Third Edition ed: Psychological Corporation 1997.

11. Laboratories ATSCoPSfCPF. ATS statement: guidelines for the six-minute walk test. Am J Respir Crit Care Med 2002; 166(1): 111-7. [PubMed: 12091180]

12. Podsiadlo D, Richardson S. The timed "Up \& Go": a test of basic functional mobility for frail elderly persons. Journal of the American Geriatrics Society 1991; 39(2): 142-8. [PubMed: 1991946]

13. Dong H, Robison LL, Leisenring WM, Martin LJ, Armstrong GT, Yasui Y. Estimating the burden of recurrent events in the presence of competing risks: the method of mean cumulative count. Am J Epidemiol 2015; 181(7): 532-40. [PubMed: 25693770]

14. Geskus RB. Cause-specific cumulative incidence estimation and the fine and gray model under both left truncation and right censoring. Biometrics 2011; 67(1): 39-49. [PubMed: 20377575]

15. Pui CH, Evans WE. A 50-year journey to cure childhood acute lymphoblastic leukemia. Semin Hematol 2013; 50(3): 185-96. [PubMed: 23953334]

16. Gibson TM, Li Z, Green DM, et al. Blood Pressure Status in Adult Survivors of Childhood Cancer: A Report from the St. Jude Lifetime Cohort Study. Cancer Epidemiol Biomarkers Prev 2017; 26(12): 1705-13. [PubMed: 29167278]

17. Whelton PK, Carey RM, Aronow WS, et al. 2017 ACC/AHA/AAPA/ABC/ACPM/AGS/ APhA/ASH/ASPC/NMA/PCNA Guideline for the Prevention, Detection, Evaluation, and Management of High Blood Pressure in Adults: A Report of the American College of Cardiology/ American Heart Association Task Force on Clinical Practice Guidelines. J Am Coll Cardiol 2017.

18. Armstrong GT, Oeffinger KC, Chen Y, et al. Modifiable risk factors and major cardiac events among adult survivors of childhood cancer. J Clin Oncol 2013; 31(29): 3673-80. [PubMed: 24002505]

19. Yeh JM, Hanmer J, Ward ZJ, et al. Chronic Conditions and Utility-Based Health-Related Quality of Life in Adult Childhood Cancer Survivors. J Natl Cancer Inst 2016; 108(9).

20. Kaste SC, Pei D, Cheng C, et al. Utility of early screening magnetic resonance imaging for extensive hip osteonecrosis in pediatric patients treated with glucocorticoids. J Clin Oncol 2015; 33(6): 610-5. [PubMed: 25605853]

21. Karol SE, Yang W, Van Driest SL, et al. Genetics of glucocorticoid-associated osteonecrosis in children with acute lymphoblastic leukemia. Blood 2015; 126(15): 1770-6. [PubMed: 26265699]

22. Ness KK, DeLany JP, Kaste SC, et al. Energy balance and fitness in adult survivors of childhood acute lymphoblastic leukemia. Blood 2015; 125(22): 3411-9. [PubMed: 25814529]

23. Braam KI, van der Torre P, Takken T, Veening MA, van Dulmen-den Broeder E, Kaspers GJ. Physical exercise training interventions for children and young adults during and after treatment for childhood cancer. Cochrane Database Syst Rev 2013; (4): CD008796. [PubMed: 23633361]

24. Scott JM, Nilsen TS, Gupta D, Jones LW. Exercise Therapy and Cardiovascular Toxicity in Cancer. Circulation 2018; 137(11): 1176-91. [PubMed: 29530893]

25. Spiegler BJ, Kennedy K, Maze R, et al. Comparison of long-term neurocognitive outcomes in young children with acute lymphoblastic leukemia treated with cranial radiation or high-dose or very high-dose intravenous methotrexate. J Clin Oncol 2006; 24(24): 3858-64. [PubMed: 16921038] 
26. Jacola LM, Krull KR, Pui CH, et al. Longitudinal Assessment of Neurocognitive Outcomes in Survivors of Childhood Acute Lymphoblastic Leukemia Treated on a Contemporary Chemotherapy Protocol. J Clin Oncol 2016; 34(11): 1239-47. [PubMed: 26858334]

27. Krull KR, Brinkman TM, Li C, et al. Neurocognitive outcomes decades after treatment for childhood acute lymphoblastic leukemia: a report from the St Jude lifetime cohort study. J Clin Oncol 2013; 31(35): 4407-15. [PubMed: 24190124]

28. Krull KR, Cheung YT, Liu W, et al. Chemotherapy Pharmacodynamics and Neuroimaging and Neurocognitive Outcomes in Long-Term Survivors of Childhood Acute Lymphoblastic Leukemia. J Clin Oncol 2016; 34(22): 2644-53. [PubMed: 27269941]

29. Schuitema I, Deprez S, Van Hecke W, et al. Accelerated aging, decreased white matter integrity, and associated neuropsychological dysfunction 25 years after pediatric lymphoid malignancies. J Clin Oncol 2013; 31(27): 3378-88. [PubMed: 23960182]

30. Ojha RP, Oancea SC, Ness KK, et al. Assessment of potential bias from non-participation in a dynamic clinical cohort of long-term childhood cancer survivors: results from the St. Jude Lifetime Cohort Study. Pediatr Blood Cancer 2013; 60(5): 856-64. [PubMed: 23024097] 


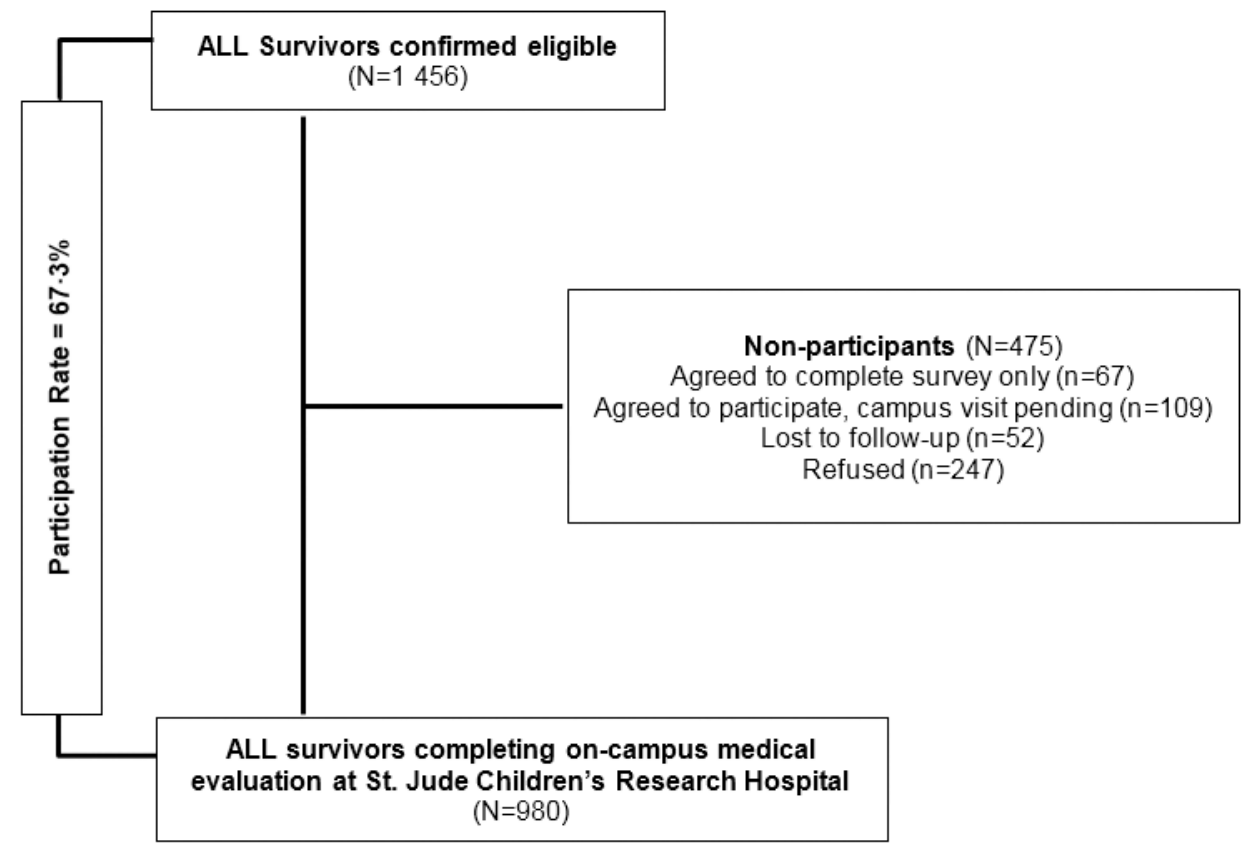

Figure 1:

Study diagram of SJLIFE population and participation in ALL analysis 
Grade 1-4

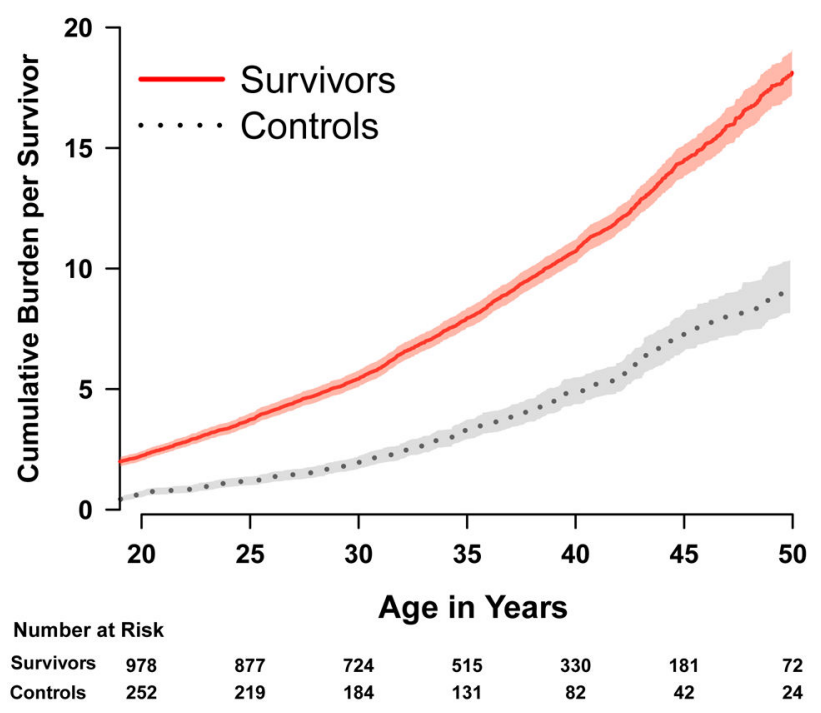

Grade 2-4

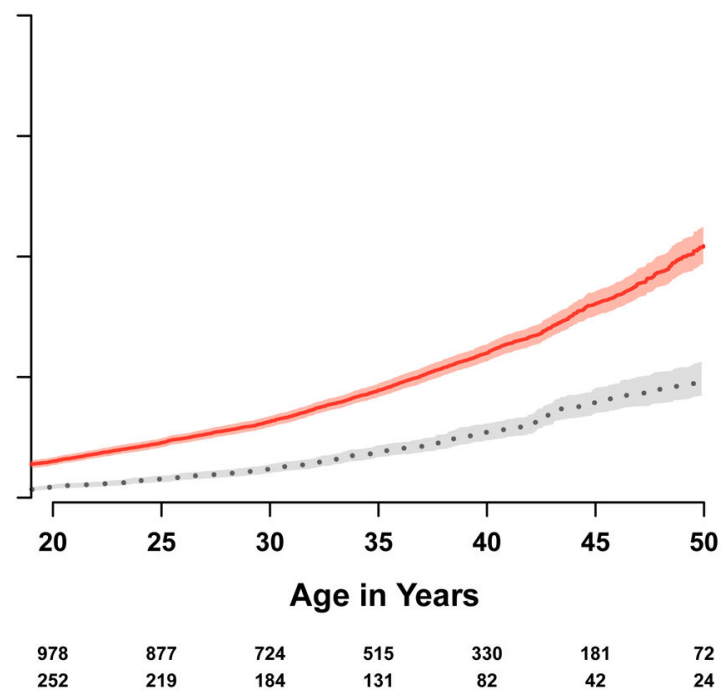

Figure 2:

Cumulative burden of health conditions

(A) Grade 1-4 events. (B) Grade 2-4 events. Among 980 survivors and 272 controls, but two survivors and 20 controls were censored before attaining age 20 years. 


\section{Grade 2-4}

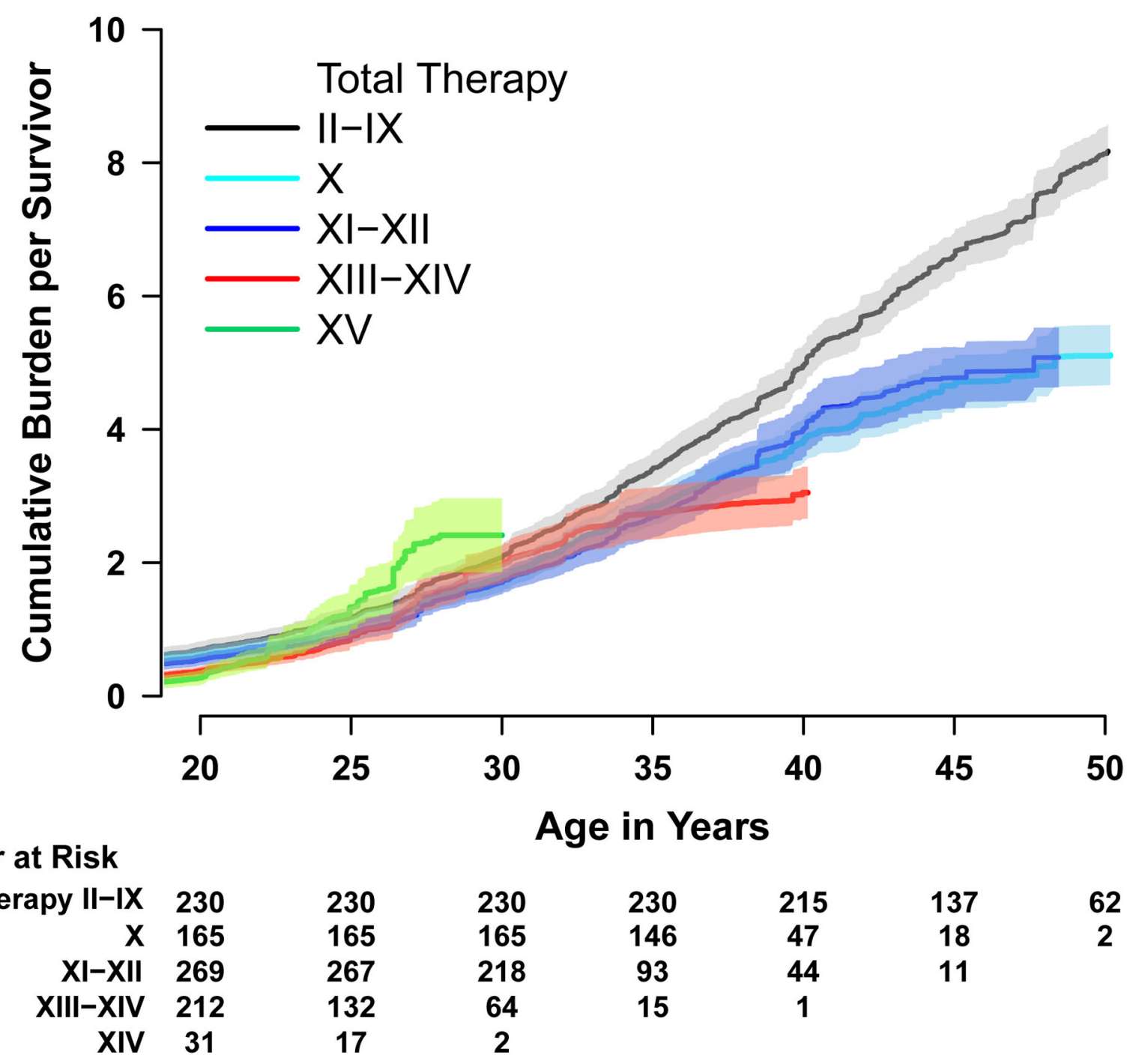

Figure 3:

Cumulative burden of grade $2-4$ health conditions in survivors by therapy protocol Among 980 survivors, 72 survivors who were treated according to a Total protocol, but who did not consent to enrolment, were excluded and one survivor in Total therapy XIII-XIV was censored before age 20 years. Thus, 907 survivors are shown here. 


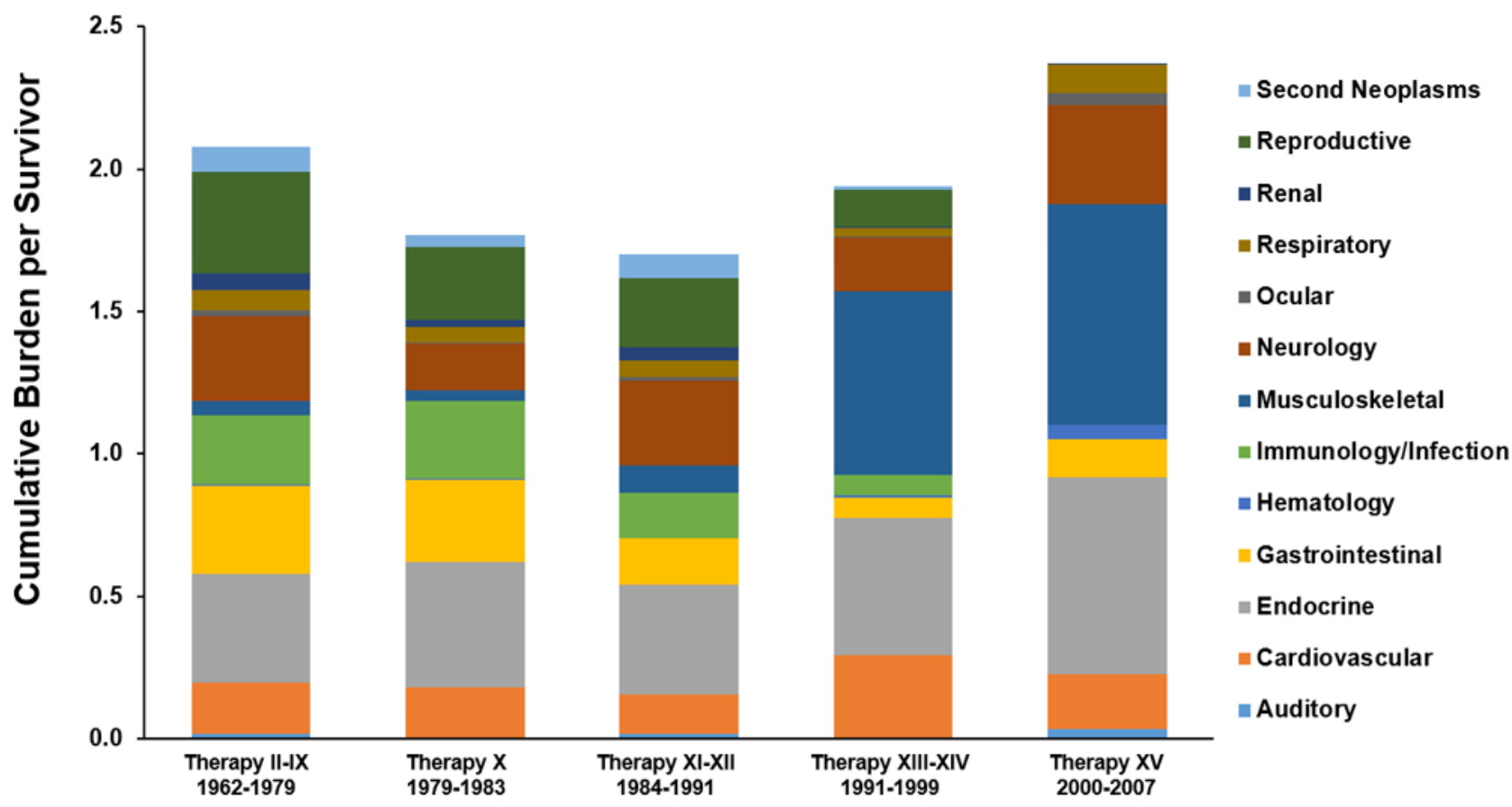

Figure 4.

Distribution of the cumulative burden of grade 2-4 health conditions among ALL survivors by Total Therapy protocol ${ }^{*}+$

* Age standardized to 30 years old

+ Excludes 72 participants who were treated according to but did not consent to enrollment on a Total protocol. 


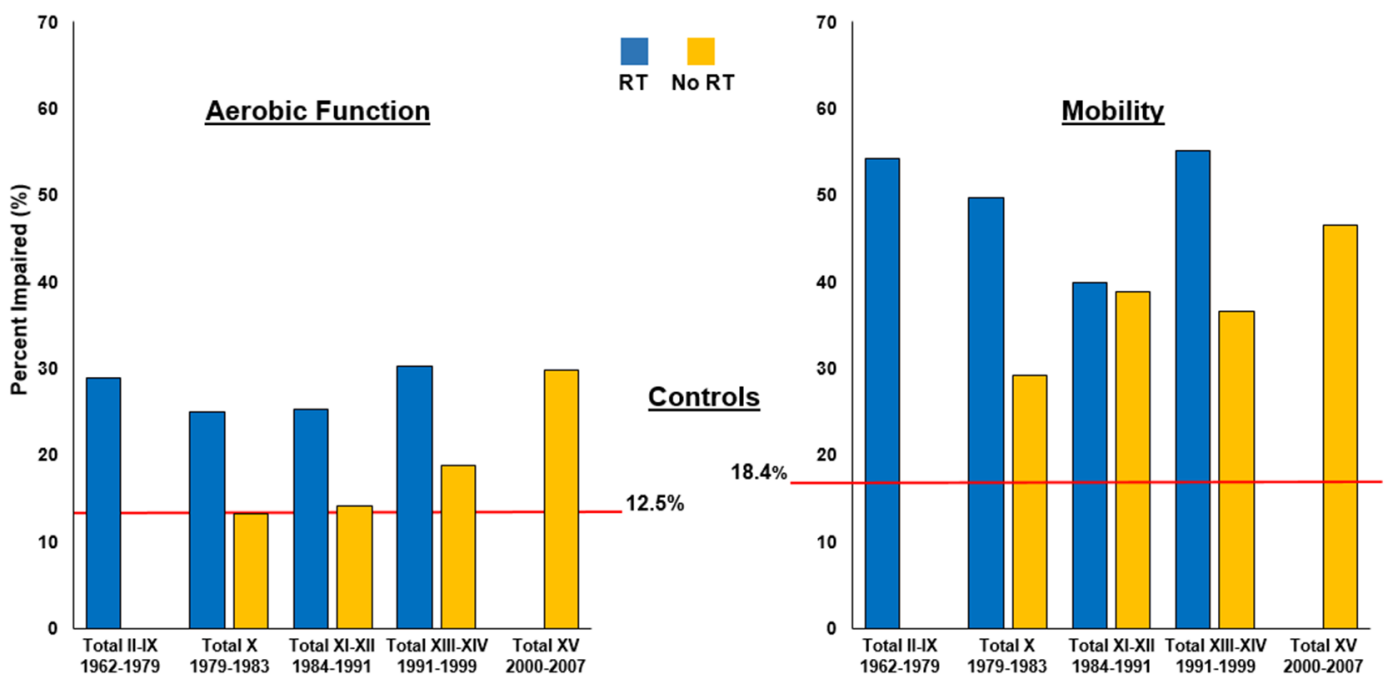

Figure 5.

Frequency of impaired aerobic dysfunction and mobility among ALL survivors by Total Therapy protocol*

* Adjusted for age at evaluation, gender, BMI, and neuropathy

+ Excludes 72 participants who were treated according to but did not consent to enrollment on a Total protocol. 


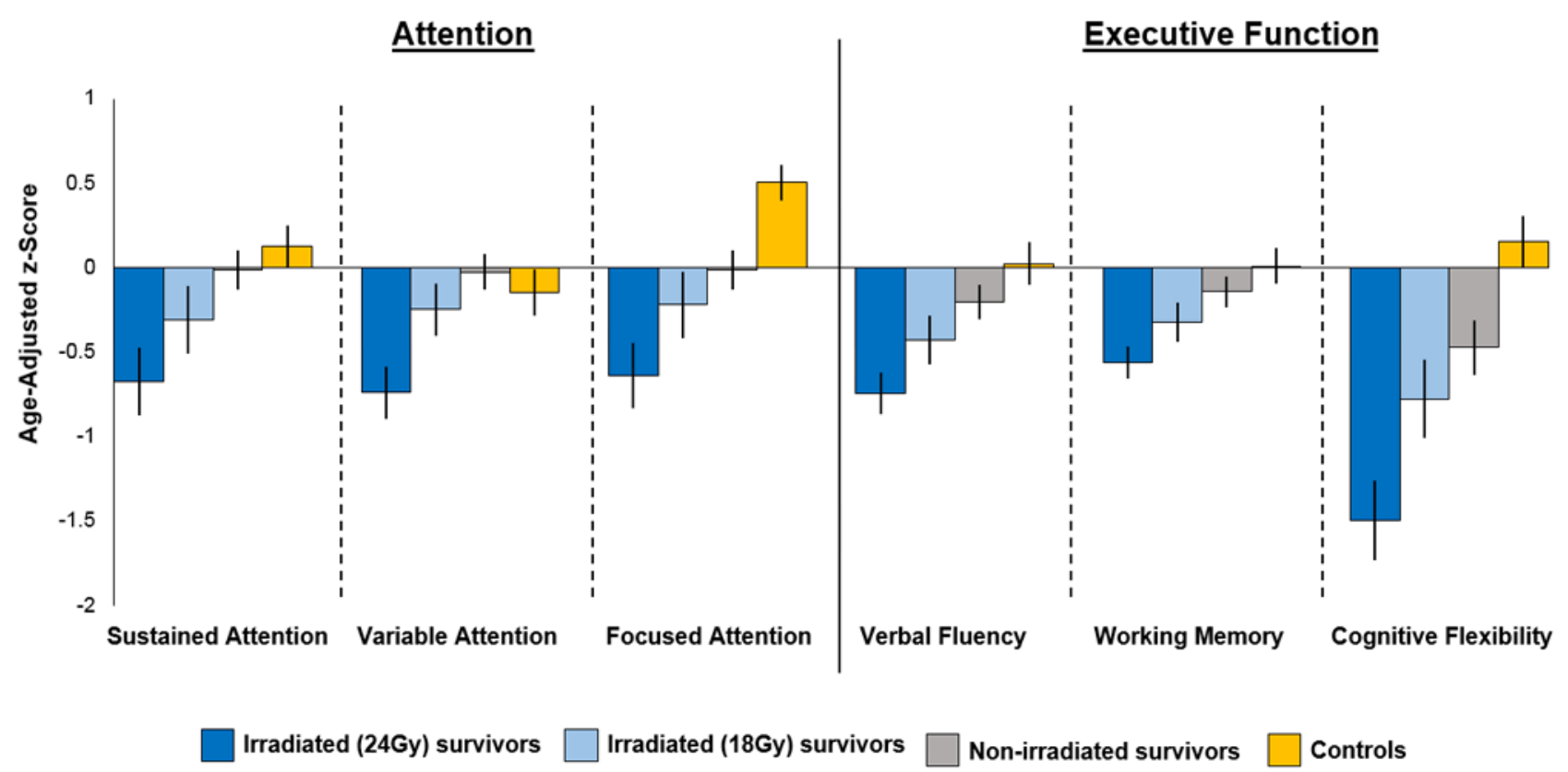

Figure 6:

Age-adjusted z-scores for attention and executive function by radiation dose and exposure in ALL survivors compared to population norms and controls 
Table 1.

Characteristics of study participants and controls

\begin{tabular}{|c|c|c|c|c|c|}
\hline & \multicolumn{2}{|c|}{$\begin{array}{l}\text { ALL Survivors } \\
(\mathbf{N}=980)\end{array}$} & \multicolumn{2}{|c|}{$\begin{array}{l}\text { Controls } \\
(\mathbf{N}=272)\end{array}$} & \multirow[b]{2}{*}{ (p) } \\
\hline & $\mathbf{N}$ & $(\%)$ & $\mathbf{N}$ & $(\%)$ & \\
\hline \multicolumn{6}{|l|}{ Gender } \\
\hline Male & 495 & $(50 \cdot 5)$ & 130 & $(47 \cdot 8)$ & \multirow{2}{*}{$\mathrm{p}=0.45$} \\
\hline Female & 485 & $(49 \cdot 5)$ & 142 & $(52 \cdot 2)$ & \\
\hline \multicolumn{6}{|l|}{$\underline{\text { Race }}$} \\
\hline White & 879 & $(89.7)$ & 238 & $(87.5)$ & \multirow{3}{*}{$\mathrm{p}=0.0054$} \\
\hline Black & 88 & $(9 \cdot 2)$ & 22 & $(8 \cdot 1)$ & \\
\hline Other & 13 & $(1 \cdot 3)$ & 12 & $(4 \cdot 4)$ & \\
\hline
\end{tabular}

Age (years)

\section{Evaluation}

18-29

30-39

$\geq 40$

$\underline{\text { Diagnosis }}$

$0-4$

5-9

$10-14$

$\geq 15$

Time since diagnosis (years)

10-19

20-29

$\geq 30$

Body Mass Index $\left(\mathrm{kg} / \mathrm{m}^{2}\right)$

Normal/underweight $(<25)$

Overweight $(25-29)$

Obese $(\geq 30)$

Education

$<12$ years

High school/GED

Vocational Training

Some college

College graduate

Post-graduate level

Employment

Full time

Part time

Unemployed

Student/Homemaker/Retired

$$
\begin{array}{cr}
\text { Median= } 35 \cdot 8 & (29.4-42 \cdot 9)^{*} \\
256 & (26 \cdot 1) \\
391 & (39 \cdot 9) \\
333 & (34 \cdot 0) \\
\text { Median=5.0 } & (3 \cdot 1-9 \cdot 1)^{*} \\
487 & (49 \cdot 7) \\
273 & (27 \cdot 9) \\
158 & (16 \cdot 1) \\
62 & (6 \cdot 3)
\end{array}
$$

$\begin{array}{ccc}\text { Median= } 35 \cdot 1 & (28 \cdot 7-42 \cdot 6)^{*} & \\ 84 & (30 \cdot 9) & \\ 102 & (37 \cdot 5) & \mathrm{p}=0 \cdot 30 \\ 86 & (31 \cdot 6) & \end{array}$

Median $=30 \cdot 0(22 \cdot 7-36 \cdot 3)^{*}$

152

338

490

$(34 \cdot 5)$

(50.0)

$\begin{array}{llrrr}276 & (28 \cdot 2) & \underline{100} & (\underline{36.8}) & \\ 269 & (27 \cdot 4) & \underline{69} & (\underline{25.4}) & \mathrm{p}=0.021 \\ 435 & (44.4) & 103 & (37.9) & \end{array}$

$\begin{array}{rrrrl}124 & (12 \cdot 7) & 11 & (4 \cdot 0) & \\ 217 & (22 \cdot 1) & 50 & (18 \cdot 4) & \\ 47 & (4 \cdot 8) & 9 & (3 \cdot 3) & \mathrm{p}<0 \cdot 0001 \\ 285 & (29 \cdot 1) & 84 & (30 \cdot 9) & \\ 238 & (24 \cdot 3) & 78 & (28 \cdot 7) & \\ 69 & (7 \cdot 0) & 40 & (14 \cdot 7) & \\ & & & & \\ 488 & (49 \cdot 8) & 172 & (63 \cdot 2) & \\ 107 & (10 \cdot 9) & 32 & (11 \cdot 8) & \\ 266 & (27 \cdot 1) & 44 & (16 \cdot 2) & \mathrm{p}<0 \cdot 0001 \\ 119 & (12 \cdot 1) & 24 & (8 \cdot 8) & \end{array}$




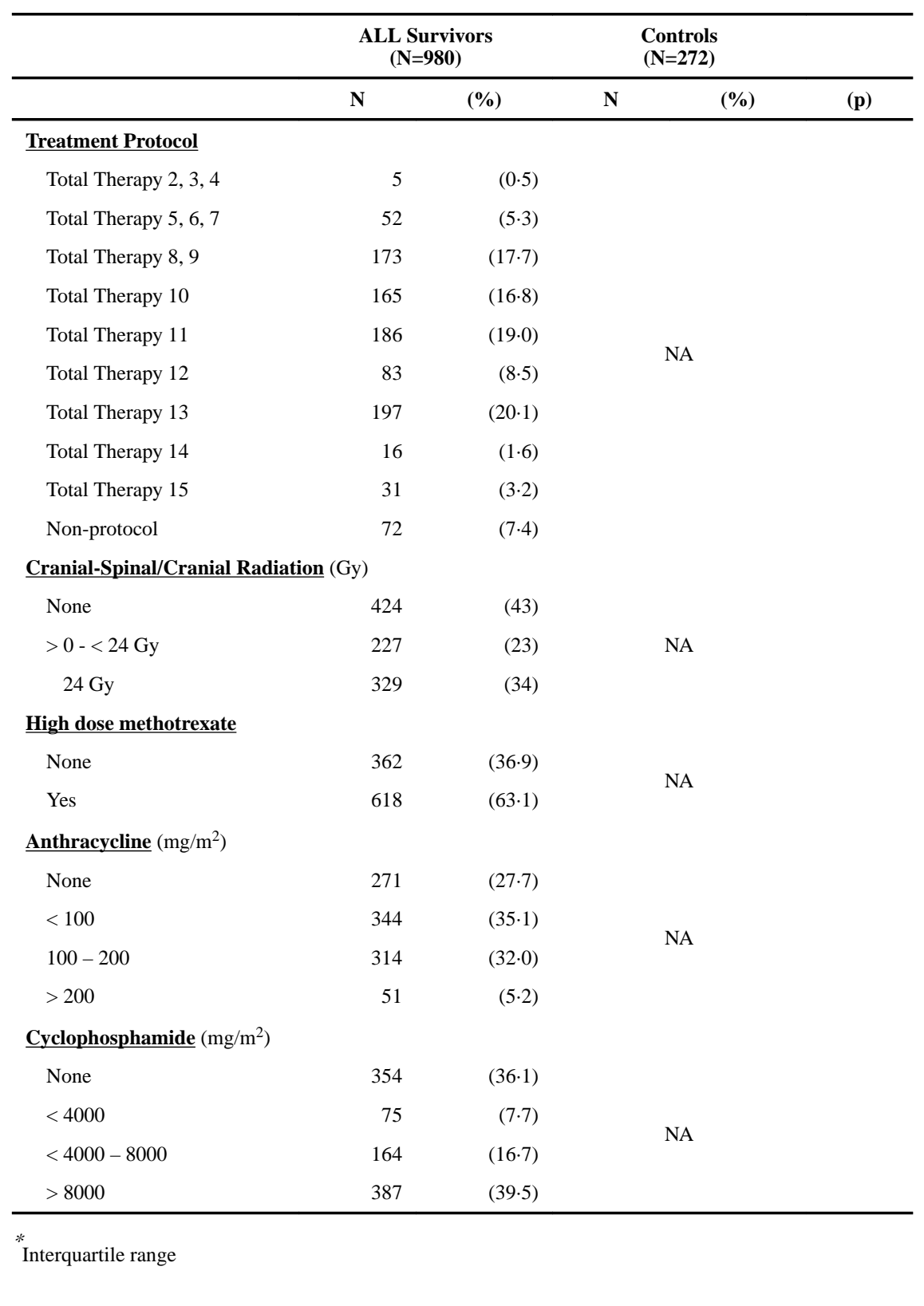




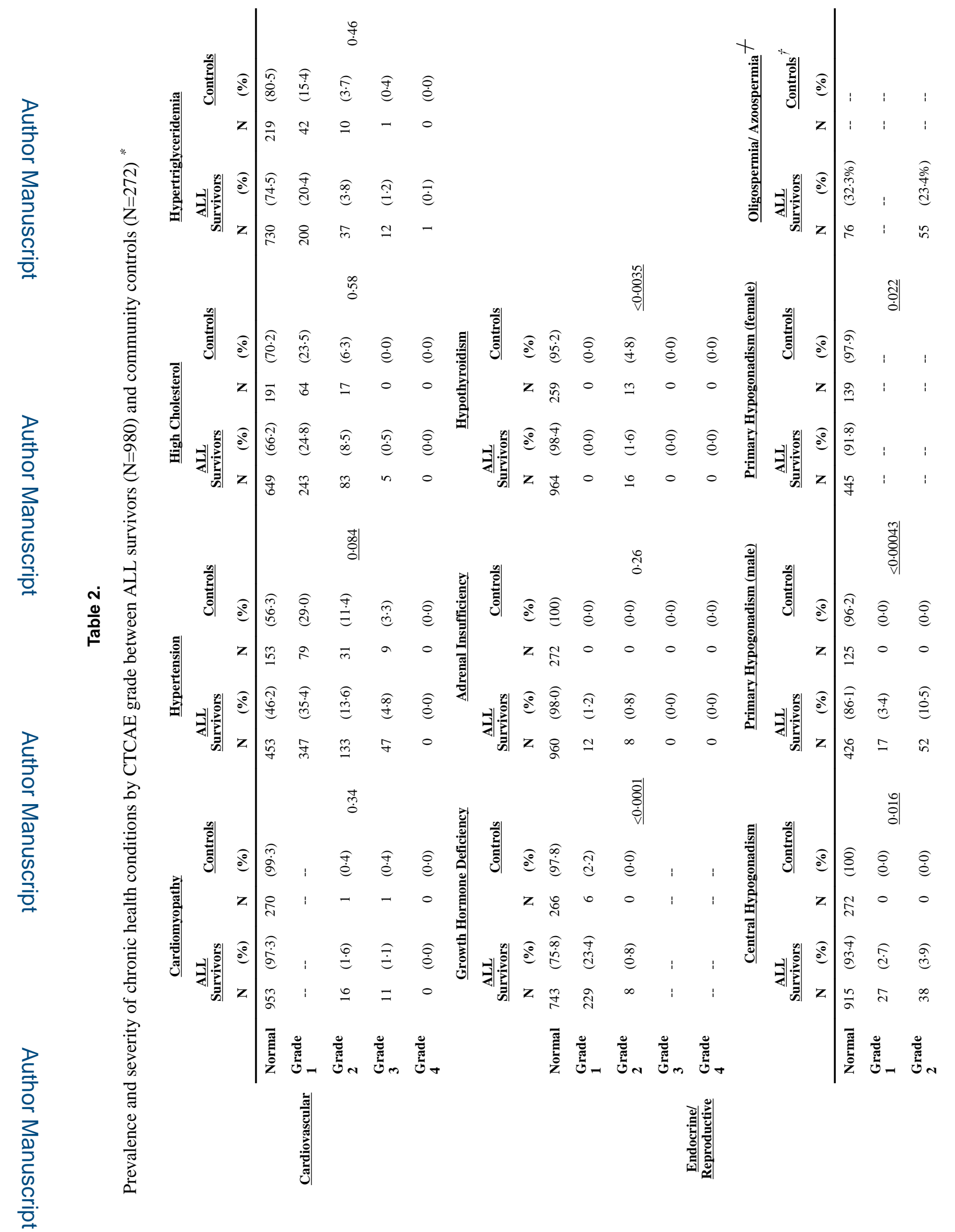

Lancet Haematol. Author manuscript; available in PMC 2020 June 01. 


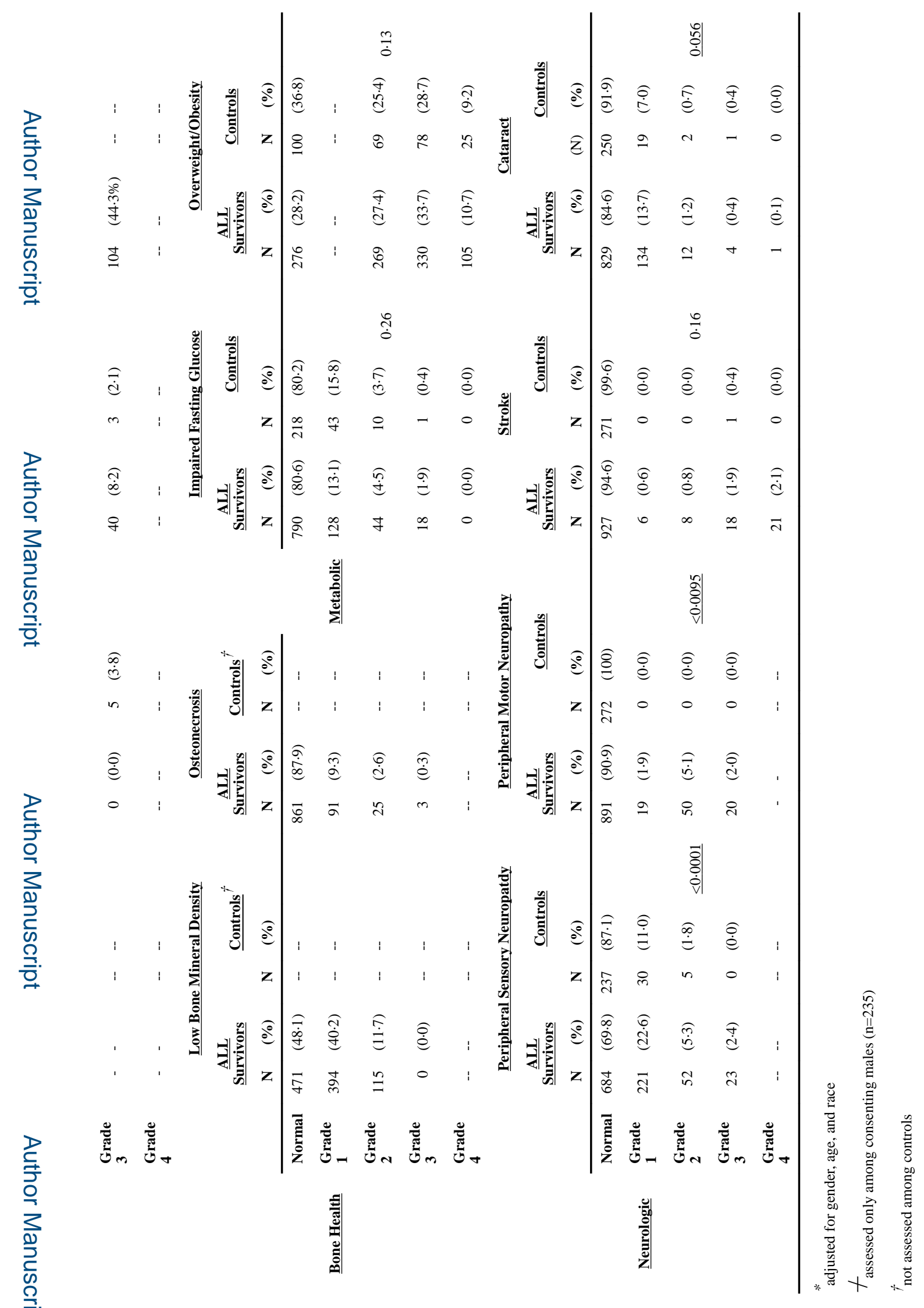

Lancet Haematol. Author manuscript; available in PMC 2020 June 01. 